CANTUA. 2016; 15:70-82

Fecha de recepción: 24.05.2016

Fecha de aceptación: 2.09.2016

\title{
Variabilidad espacio temporal de la comunidad bentónica de la Laguna de Piuray - Cusco
}

\section{Temporary space variability of the benthonic community of Laguna de Piuray - Cusco}

\author{
Katherine M. Cruz-Fernández Baca ${ }^{1}$, Luciano J. Cruz-Miranda ${ }^{1}$ \\ ${ }^{1}$ Escuela Profesional de Biología. Facultad de Ciencias. Universidad Nacional de San Antonio \\ Abad del Cusco Cusco, Perú.
}

\section{RESUMEN}

Los organismos bentónicos en los ecosistemas acuáticos participan en la transferencia de energía dentro de la cadena trófica, actúan como bioindicadores y permiten evaluar el grado de perturbación en diferentes épocas y espacios de la cubeta de la laguna, se determinó la composición, abundancia y diversidad de la comunidad bentónica de la laguna de Piuray, con la finalidad de evaluar su variabilidad espacio-temporal, muestreando con una draga Eckman con área de cobertura de $0.04 \mathrm{~m} 2$ y volumen de $0.0033 \mathrm{~m} 3$, a profundidades de $1,1.5$ y 2 metros, en tres zonas de la laguna; se registró 7508 individuos, distribuidos en 9 familias y 5 órdenes (Gasterópoda, Veneroida, Oligochaeta, Díptera e Hirudinea). El $44.40 \%$ de familias corresponden al Orden Gasterópoda, 22.22 \% al Orden Díptera y $11.11 \%$ a cada orden Veneroida, Oligochaeta e Hirudinea. La mayor densidad se registra cerca a la orilla, descendiendo con la profundidad; con la familia Planorbidae con 58215 individuos $/ \mathrm{m}^{3}$ y la menor densidad se registró con la familia Ceratopogonidae con 135 individuos $/ \mathrm{m}^{3}$, mientras que las familias con mayor abundancia fueron Planorbidae y Tubificidae. La diversidad alfa mediante el Índice de Shannon es de 0 1.45 y de Simpson de $0-0.7$ mostrando una baja diversidad; la diversidad beta mediante el Índice de Jaccard registra similaridad entre 15 y 100\%; mientras que el Índice de Morisita registra homogeneidad entre 8 y $100 \%$, indicando que existe variación en el espacio y tiempo de la 
comunidad bentónica de la laguna de Piuray en los meses evaluados.

PALABRAS CLAVE: Comunidad bentónica, variación espacial, variación temporal, diversidad.

\begin{abstract}
Benthic organisms in aquatic ecosystems participate in the transfer of energy within the trophic chain, they act as bioindicators to evaluate the degree of disturbance in different times and spaces of the lagoon basin, we determined the composition, abundance and diversity of the benthic community of the Piuray lagoon, in order to evaluate its spatio-temporal variability, sampling with an Eckman dredge with a coverage area of $0.04 \mathrm{~m} 2$ and a volume of $0.0033 \mathrm{~m} 3$, at depths of 1, 1.5 and 2 meters, in three areas of the lagoon; 7508 individuals were registered, distributed in 9 families and 5 orders (Gastropoda, Veneroida, Oligochaeta, Diptera and Hirudinea). $44.40 \%$ of families correspond to the Gastropoda Order, $22.22 \%$ to the Diptera Order and $11.11 \%$ to each Veneroida, Oligochaeta and Hirudinea order. The highest density is recorded near the shore, decreasing with depth; registering in the family Planorbidae with 58215 organisms $/ \mathrm{m}^{3}$ and the lowest density is the family Ceratopogonidae con 135 organisms $/ \mathrm{m}^{3}$; the most abundant families were Planorbidae and Tubificidae. The alpha diversity through the Shannon Index is $0-1.45$ and Simpson's $0-0.7$ showing a low diversity; the beta diversity by means of the Jaccard Index registers similarity between 15 and 100\%; while the Morisita Index registers homogeneity between 8 and $100 \%$, indicating that there is variation in space and time of the benthic community of the Piuray lagoon in the months evaluated.
\end{abstract}

KEY WORDS: benthic fauna, spatial variation, temporal variation, diversity.

\title{
INTRODUCCIÓN
}

Los ecosistemas acuáticos están constituidos por una variedad de hábitats que albergan comunidades de organismos, que influyen en la ecología del ambiente. Dentro de estas comunidades se encuentran los organismos bentónicos, los cuales viven en relación con el fondo lacustre de los cuerpos lénticos como la laguna de Piuray.

Alrededor de la laguna de Piuray, se encuentran asentados 11 centros poblados, que desarrollan actividades agrícolas, pecuarias; así como actividades domésticas (Centro de Educación y 
Comunicación Guaman Poma de Ayala, 2013). En la laguna de Piuray, el crecimiento e incremento de la densidad de la flora ribereña, así como su descomposición perturban dicho ecosistema, incrementando los sedimentos en el fondo de la laguna. Estas perturbaciones influyen en la composición de las comunidades bentónicas de la laguna, generando variaciones espacio - temporales.

La laguna de Piuray es un ecosistema lacustre de importancia para la ciudad del Cusco, no sólo por ser uno de los recursos hídricos de servicio para la ciudad, sino también por ser un refugio para la diversidad biológica, en especial para aves silvestres y migratorias.

El crecimiento poblacional en la ciudad del Cusco ha incrementado la demanda de consumo de agua dulce, que sumado al impacto que generan las actividades humanas asentadas sobre este ecosistema léntico alteran la calidad de agua, por lo que es importante conocer el grado de perturbación, a través de la composición y distribución de los organismos bentónicos.

En el presente estudio se aplicó metodologías de identificación taxonómica mediante claves, se cuantifico la densidad y abundancia y para la diversidad alfa y beta los índices de Shannon, Simpson, Jaccard y Morisita.

Por la importancia de la laguna de Piuray, la investigación referente al estudio de la variabilidad espacio- temporal de la comunidad bentónica permitirá determinar el grado de perturbación que pueda tener el cuerpo léntico y coadyuvar al manejo del ecosistema lacustre de la laguna de Piuray.

\section{MATERIALES Y MÉTODOS}

Área de Estudio

La laguna de Piuray se encuentra en la cuenca endorreica de Piuray Ccorimarca, dentro de la cuenca Urubamba, se sitúa a $30 \mathrm{Km}$ de la ciudad del Cusco, en el distrito de Chinchero, Provincia de Urubamba, Región Cusco, a una altitud de 3.750 m.s.n.m. y con una superficie de 9,578.29 ha. Entre las coordenadas UTM: 820000 - 822500 E y $8514000-8515500 \mathrm{~N}$ (figura $1)$. 


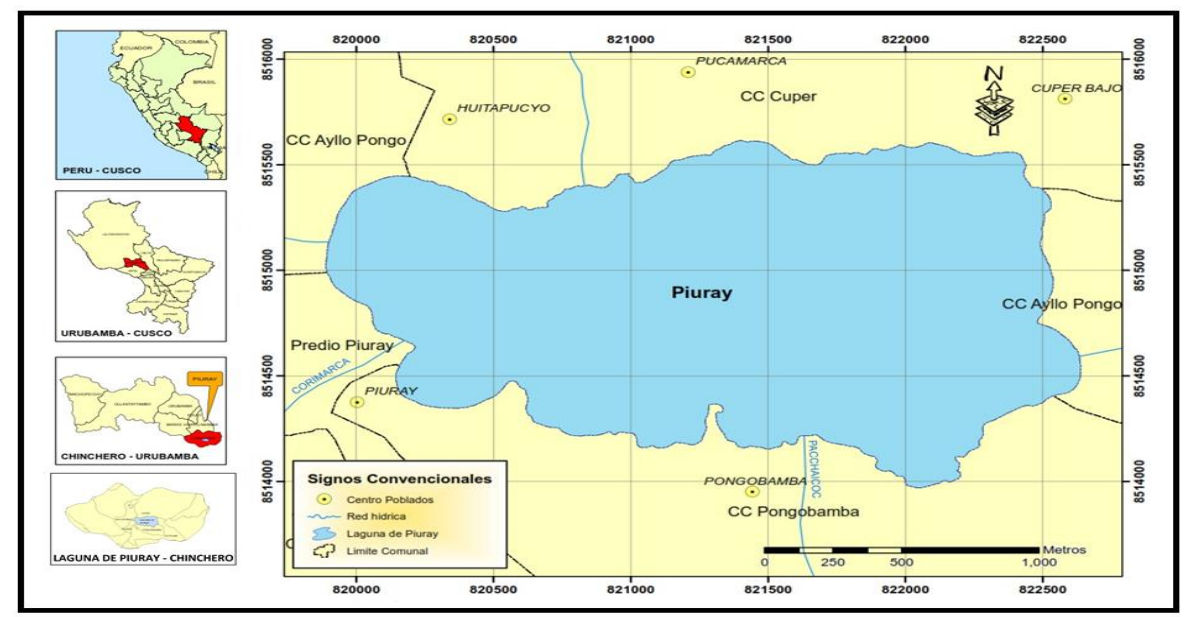

Figura1. Ubicación política y geográfica de la laguna de Piuray. Fuente: Rosas y Miranda, 2014.

La cuenca de la laguna de Piuray, tiene como principales fuentes de alimentación a riachuelos como Ravilchaca, Pongobamba, Ocotuan, Huila Huila y el manantial Maychu siendo el único efluente el punto de captación de Seda-Cusco (Rosas y Miranda, 2014). El área de estudio se encuentra en la zona de vida: Bosque húmedo - Montano Subtropical (bh-MS), comprendida entre 3350 a 3900 msnm., con precipitación anual de 700 a 850 mm, y temperatura media anual de 8.5 a $10^{\circ} \mathrm{C}$. (Centro de Educación y Comunicación Guaman Poma de Ayala, 2013).

Considerando la presencia de poblaciones asentadas, el desarrollo de actividades antrópicas, la plataforma de la laguna y la accesibilidad, se determinaron 03 zonas de estudio; cada una de ellas representada por 03 puntos de muestreo, haciendo un total de 09 puntos de muestreo, georreferenciados con GPS (GARMIN) modelo Colorado 400t (figura 2).

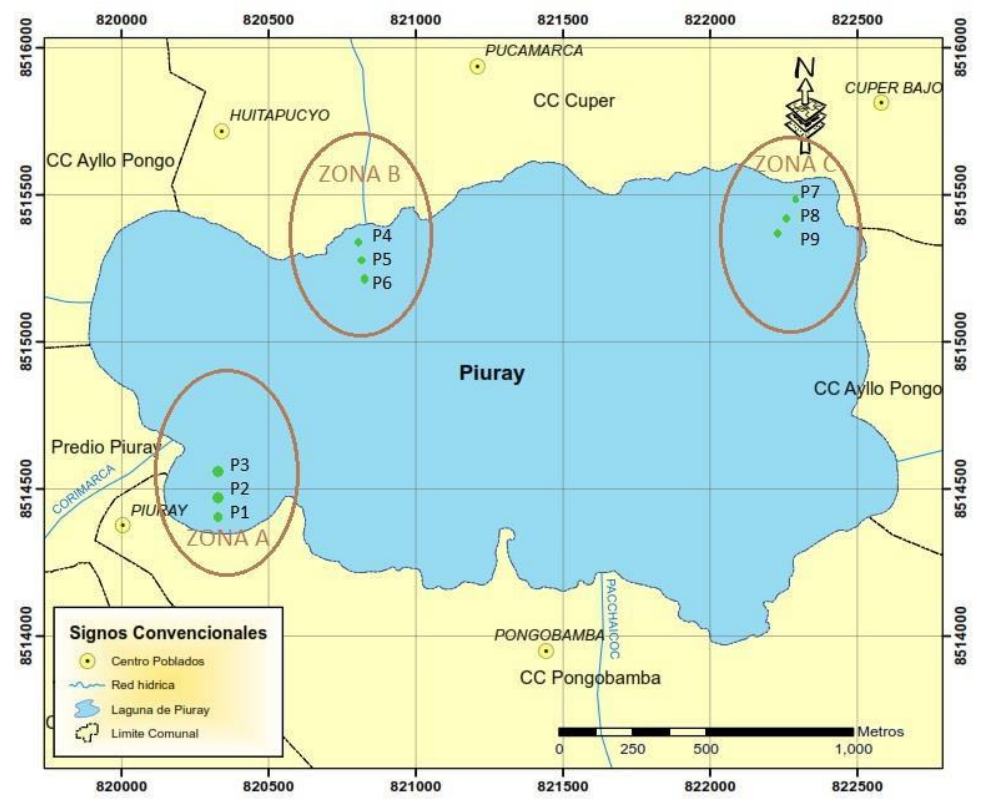

Figura 2. Ubicación de zonas y puntos de muestreo. 
Las muestras fueron colectadas en el período de mayo a julio, a $1 \mathrm{~m} ., 1.5 \mathrm{~m}$. y $2 \mathrm{~m}$. de profundidades preestablecidas, mediante el uso de una draga Eckman con un área de cobertura de $0.04 \mathrm{~m}^{2}$ y volumen de $0.0033 \mathrm{~m}^{3}$; para luego ser colocadas en bolsas debidamente etiquetadas y rotuladas.

Una vez extraída la muestra, se procede a la limpieza de las muestras, para luego ser conservadas en frascos con alcohol al 70\%, rotulando y etiquetando con la siguiente información:

Nombre de la investigación

$\begin{array}{ll}- & \text { Nombre de la laguna } \\ \text { - } & \text { Localidad } \\ \text { - } & \text { Fecha } \\ \text { - } & \text { Número de muestra } \\ \text { - } & \text { Tipo de Conservante }\end{array}$

Para la evaluación de la composición de la comunidad bentónica se aplicó el método de identificación taxonómica para lo cual las muestras se colocaron en una bandeja blanca para separar los individuos con la ayuda de pinzas y estiletes, se observó en un estereoscopio para su determinación, mediante el uso de claves taxonómicas propuestas por Oscoz, J.; Galicia, D.; Miranda, R. (2011) y Clifford H. (1991).

La determinación de la densidad se realizó mediante el conteo de los organismos bentónicos, determinando el número de individuos por familia por metro cúbico; y para la abundancia se calculó la proporción de los individuos de una familia con respecto al total de individuos de la comunidad.

La diversidad de la comunidad bentónica se calculó usando los Índices de Shannon - Weaver y Simpson para la diversidad Alfa (Roldán, 1999) y los Índices de Morisita y Jaccard para la diversidad Beta.

\section{RESULTADOS}

Se colectaron 7508 individuos distribuidos en 9 familias y 5 órdenes (Gasterópoda, Veneroida, Oligochaeta, Díptera e Hirudinea) en la comunidad bentónica.

La comunidad bentónica está conformada por el $44 \%$ del orden Gasterópodo representado con 4 familias, seguido de Díptera con $22.22 \%$ con 2 familias y los órdenes Veneroida, Oligochaeta e Hirudinea con $11.11 \%$ cada una conformadas con una familia (tabla 1). 
Tabla 1.

Composición de la Comunidad Bentónica de la laguna de Piuray

\begin{tabular}{lcc}
\hline Orden & Familia & Porcentaje Familias \\
\hline Gasterópoda & Acroloxidae & $44.44 \%$ \\
& Lymnaeidae & \\
& Physidae & \\
& Planorbidae & \\
Veneroida & Sphaeriidae & $11.11 \%$ \\
Oligochaeta & Tubificidae & $11.11 \%$ \\
Díptera & Ceratopogonidae & $22.22 \%$ \\
& Chironomidae & \\
Hirudinea & Glossiphoniidae & $11.11 \%$ \\
\hline
\end{tabular}

La presencia de la comunidad bentónica en el periodo de estudio es variable: así Acroloxidae y Planorbidae se registran en todo el periodo de muestreo y en las 3 zonas, siendo familias constantes, mientras que la presencia de las familias Lymnaeidae, Physidae, Sphaeriidae, Tubificidae, Ceratopogonidae, Chironomidae y Glossiphoniidae, es variable en el tiempo y el espacio de la laguna de Piuray (tabla 2).

Tabla 2.

Variación espacio-temporal de la Comunidad Bentónica de la laguna de Piuray

\begin{tabular}{|c|c|c|c|c|c|c|c|c|c|c|}
\hline \multirow{3}{*}{ Orden } & \multirow{3}{*}{ Familia } & \multicolumn{9}{|c|}{ Mes } \\
\hline & & \multicolumn{3}{|c|}{ Mayo } & \multicolumn{3}{|c|}{ Junio } & \multicolumn{3}{|c|}{ Julio } \\
\hline & & $\begin{array}{c}\text { Zona } \\
\text { A }\end{array}$ & $\begin{array}{c}\text { Zona } \\
\text { B }\end{array}$ & $\begin{array}{c}\text { Zona } \\
\text { C }\end{array}$ & $\begin{array}{c}\text { Zona } \\
\text { A }\end{array}$ & $\begin{array}{c}\text { Zona } \\
\text { B }\end{array}$ & $\begin{array}{c}\text { Zona } \\
\text { C }\end{array}$ & $\begin{array}{c}\text { Zona } \\
\text { A }\end{array}$ & $\begin{array}{c}\text { Zona } \\
\text { B }\end{array}$ & $\begin{array}{c}\text { Zona } \\
\text { C }\end{array}$ \\
\hline \multirow[t]{4}{*}{ Gasterópoda } & Acroloxidae & $\mathrm{x}$ & $\mathrm{x}$ & $\mathrm{x}$ & $\mathrm{x}$ & $\mathrm{x}$ & $\mathrm{x}$ & $\mathrm{x}$ & $\mathrm{x}$ & $\mathrm{x}$ \\
\hline & Lymnaeidae & & & & & $\mathrm{x}$ & $\mathrm{x}$ & $\mathrm{x}$ & $\mathrm{x}$ & $\mathrm{x}$ \\
\hline & Physidae & & $\mathrm{x}$ & $\mathrm{x}$ & $\mathrm{x}$ & $\mathrm{x}$ & $\mathrm{x}$ & $\mathrm{x}$ & $\mathrm{x}$ & $\mathrm{x}$ \\
\hline & Planorbidae & $\mathrm{x}$ & $\mathrm{x}$ & $\mathrm{x}$ & $\mathrm{x}$ & $\mathrm{x}$ & $\mathrm{x}$ & $\mathrm{x}$ & $\mathrm{x}$ & $\mathrm{x}$ \\
\hline Veneroida & Sphaeriidae & & & & & $\mathrm{x}$ & & $\mathrm{x}$ & & $\mathrm{x}$ \\
\hline Oligochaeta & Tubificidae & $\mathrm{x}$ & $\mathrm{x}$ & $\mathrm{x}$ & & $\mathrm{x}$ & $\mathrm{x}$ & $\mathrm{x}$ & $\mathrm{x}$ & $\mathrm{x}$ \\
\hline \multirow[t]{2}{*}{ Díptera } & Ceratopogonidae & & & & & $\mathrm{x}$ & & & & $\mathrm{x}$ \\
\hline & Chironomidae & & & & & $\mathrm{x}$ & $\mathrm{x}$ & $\mathrm{x}$ & $\mathrm{x}$ & $\mathrm{x}$ \\
\hline Hirudinea & Glossiphoniidae & & $\mathrm{x}$ & $\mathrm{x}$ & & $\mathrm{x}$ & $\mathrm{x}$ & $\mathrm{x}$ & & $\mathrm{x}$ \\
\hline
\end{tabular}


La mayor densidad registra la familia Planorbidae con 58215 individuos $/ \mathrm{m}^{3}$ y una menor densidad la familia Ceratopogonidae con 135 individuos $/ \mathrm{m}^{3}$, mientras que las familias con mayor abundancia fueron Planorbidae y Tubificidae (figura 3).

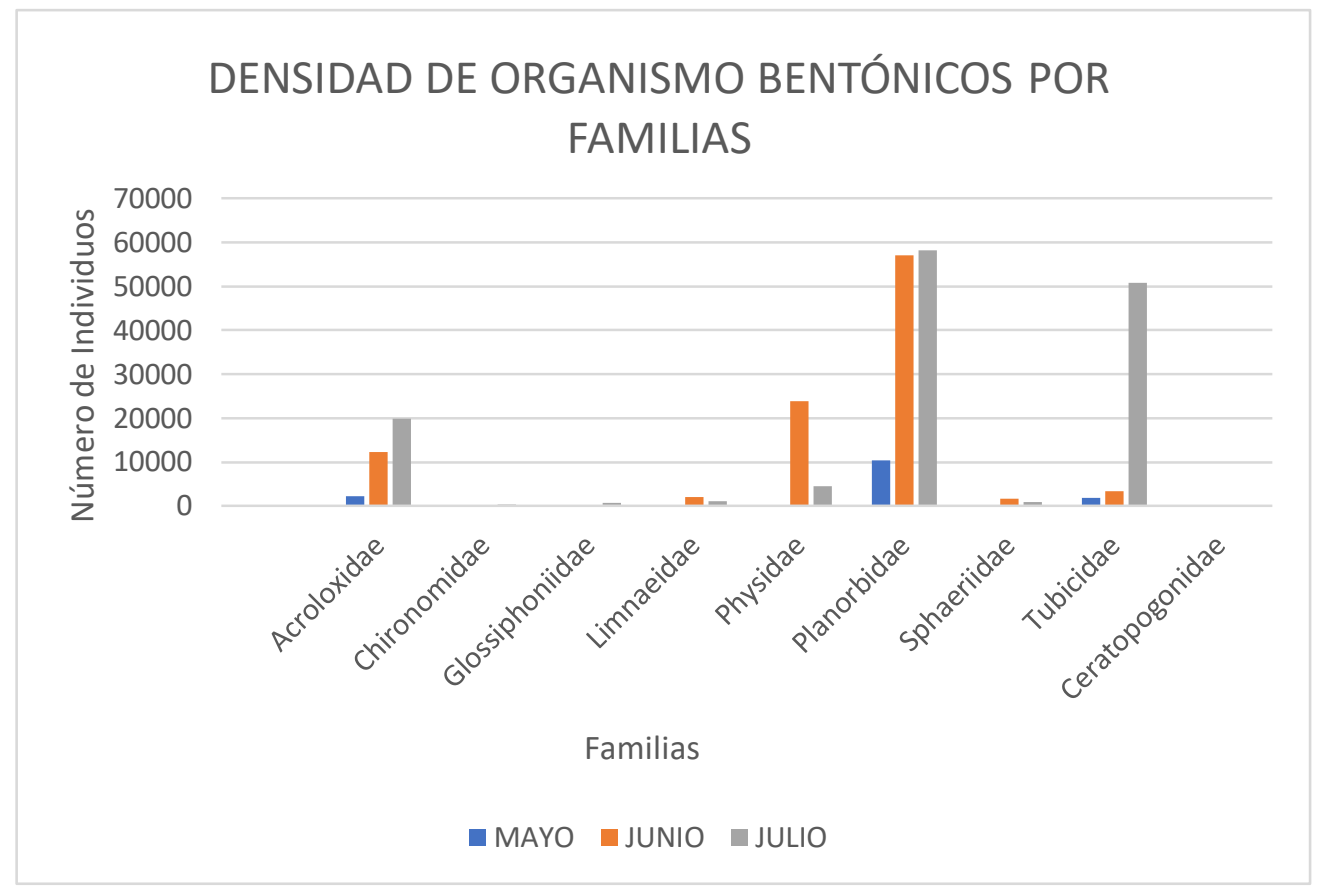

Figura 3. Densidad de la comunidad bentónica de la laguna de Piuray (indv/ $\mathrm{m}^{3}$ ).

La diversidad Alfa muestra que en el mes de mayo los valores más altos fueron para el índice de Simpson 0.5313 y para el índice de Shannon 0.9003, ambos valores ubicados en el Punto 8 de la zona C. En el mes de junio el valor más alto para Simpson es 0.7041 y para Shannon 1.272, ambos valores ubicados en el Punto 7 de la zona $\mathrm{C}$ y en el mes de julio el valor más alto para el índice de Simpson es 0.6904 y para el índice de Shannon 1.447, ambos valores ubicados en el Punto 3 de la zona A.

La diversidad beta, mediante el índice de Jaccard para el mes de mayo como se observa en la figura 4 indica una similitud cercana al 100\% entre los puntos 3 y 6 , en $80 \%$ entre los puntos 4 y 5 y el resto de los puntos presentan disimilitud. Por otro lado, el índice de Morisita para mayo muestra una homogeneidad entre el punto 3 y 6 cercana al $100 \%$ y entre los puntos 4 y 7 alcanza un $98 \%$ de similitud (Figura 4). 

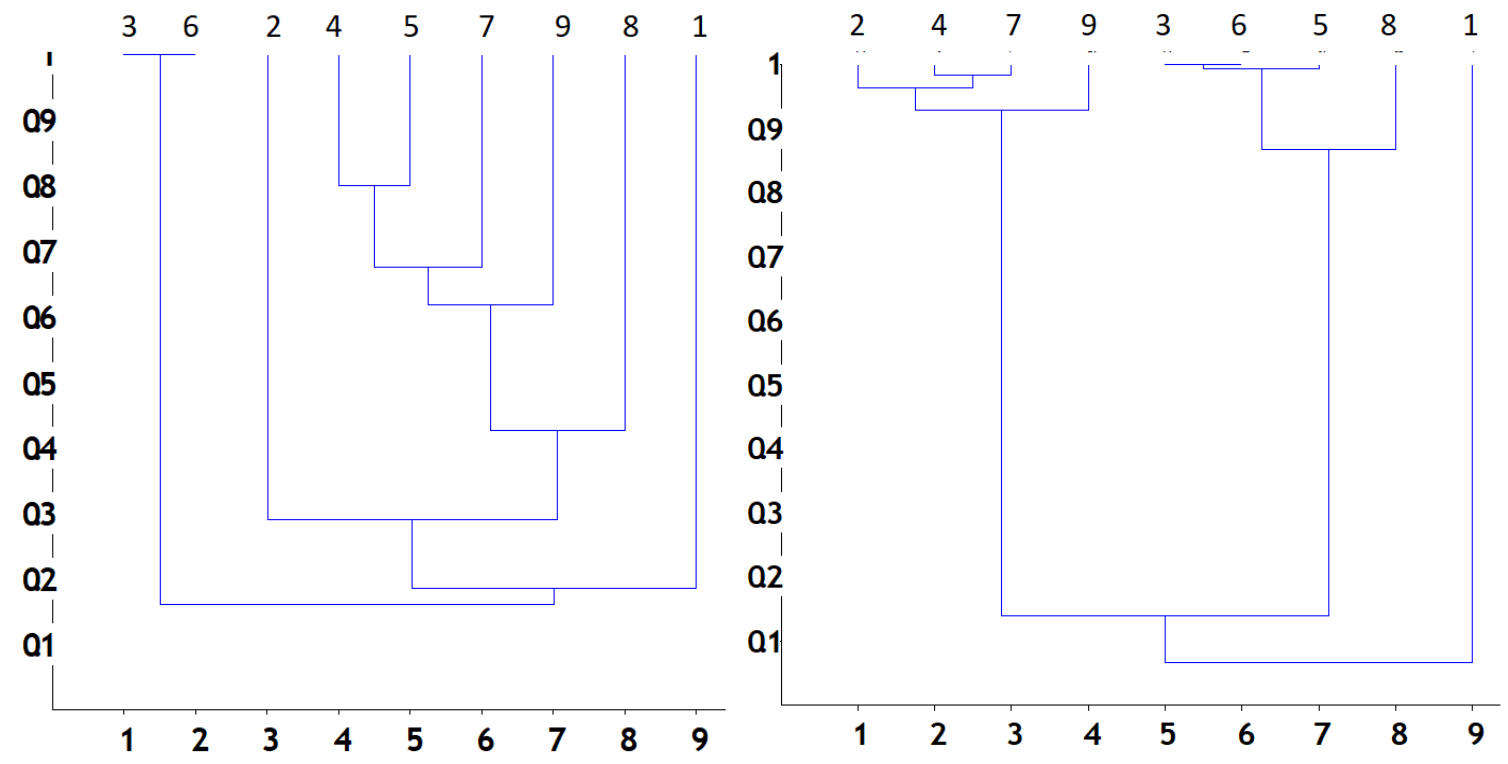

Figura 4. Diversidad Beta (Índice de Jaccard - Índice de Morisita) - mayo.

En junio el índice de Jaccard (Figura 5) muestra una similitud cercana al 100\% entre los puntos 1 y 3 y en $80 \%$ entre los puntos 4 y 7 , el resto de puntos presenta disimilitud. El índice de Morisita para junio muestra una homogeneidad entre el punto 8 y 9 cercana al $97 \%$ y entre los puntos 3 y 4 alcanza un $96 \%$ de similitud el resto de los puntos son heterogéneos (figura 5).

Índice Jaccard

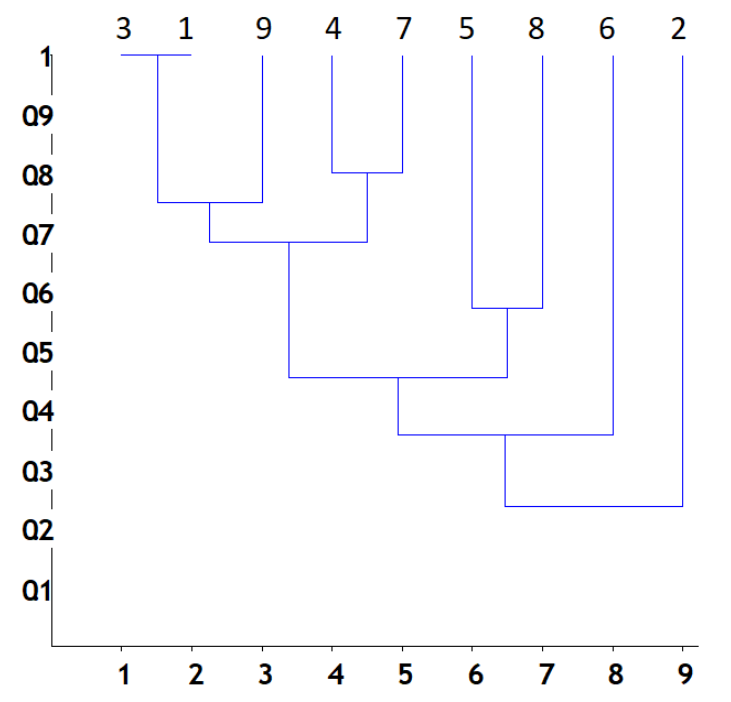

Índice Morisita

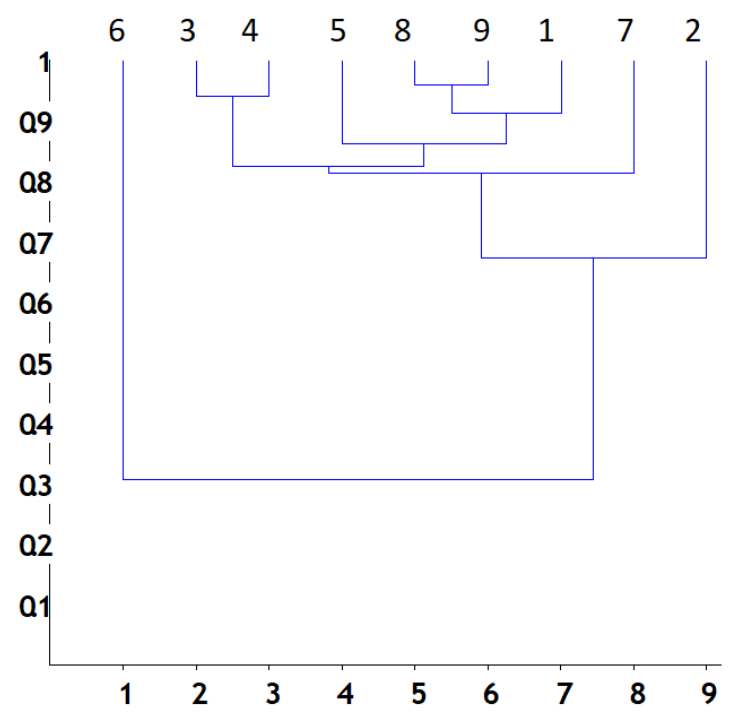

Figura 5. Diversidad Beta (Índice de Jaccard - Índice de Morisita) - junio. 
En el mes de julio el índice de Jaccard (Figura 6) muestra una similitud cercana al 100\% entre los puntos 1 y 5 y en $82 \%$ entre los puntos 4 y 6 , el resto de los puntos presenta disimilitud. El índice de Morisita para julio muestra una homogeneidad entre el punto 7 y 9 cercana al 98\%; entre los puntos 2 y 4 alcanza un $95 \%$ de similitud y entre los puntos 5 y 6 un $92 \%$. el resto de los puntos son heterogéneos (figura 6).

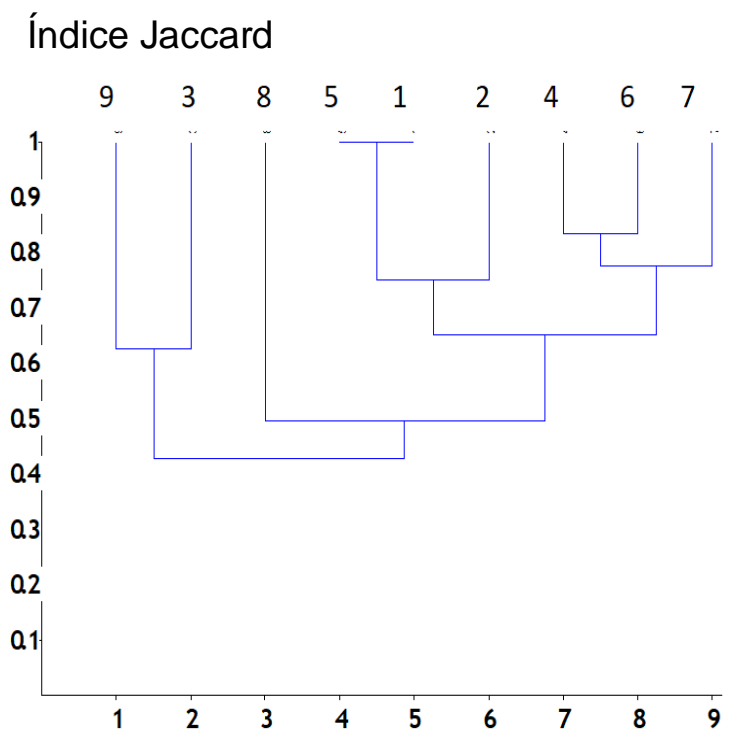

Índice Morisita

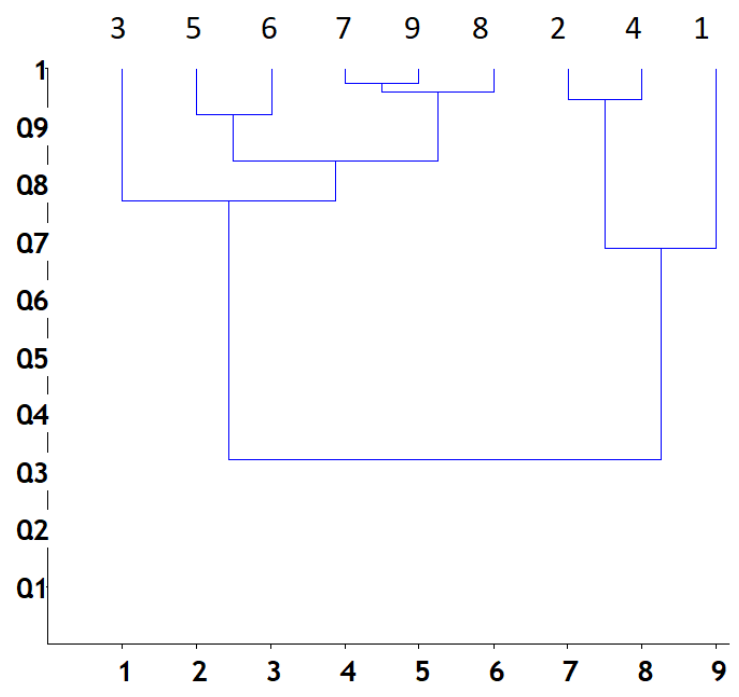

Figura 6. Diversidad Beta (Índice de Jaccard - Índice de Morisita) - julio.

En el anexo 1 se muestran fotos tomadas durante el desarrollo del presenta tabajho de investigación

\section{DISCUSIÓN}

Del análisis de 27 muestras procedentes de profundidades pre establecidas en 9 puntos de muestreo pertenecientes a 3 zonas de la laguna de Piuray, durante los tres meses de estudio, el Orden Gasterópoda con las familias Planorbidae, Physidae, Acroloxidae y Lymnaeidae tiene una presencia constante a lo largo del período de muestreo la familia Tubificidae del Orden Oligochaeta, la familia Chironomidae del Orden Díptera y la familia Glossiphoniidae del Orden Hirudinea, son variables en el tiempo y el espacio de la laguna de Piuray.

La densidad relativa de las familias bentónicas varía en los diferentes meses, así como en las zonas y profundidades de muestreo, como manifiesta Karr (1991). 
El índice de Shannon varía de 0 a 1.447 y el de Simpson varía de 0 a 0.7041 indicando una baja diversidad y alta dominancia; respecto a la variación espacio temporal, la zona "A" posee una mayor diversidad respecto a las zonas B y C, esta variación de la diversidad se incrementó al transcurrir el período de estudio, coincidiendo con la recuperación de la laguna del fenómeno de San Juan ocurrido en el mes de mayo.

\section{CONCLUSIONES}

La comunidad bentónica de la laguna de Piuray para el periodo de investigación, está representada por 05 Órdenes con 09 familias, entre los cuales el orden mejor representado son los Gasterópodos con las familias Planorbidae, Physidae, Acroloxidae y Lymnaeidae; seguido del Orden Díptera con 02 familias: Ceratopogonidae y Chironomidae, el Orden Hirudinea con la familia Glossiphoniidae, el Orden Oligochaeta con la familia Tubificidae y el Orden Veneroida con la familia Sphaeriidae. La mayor densidad la presento la familia Planorbidae con 58215 individuos/m3 y la menor densidad la familia Ceratopogonidae con 135 individuos/m3, mientras que las familias con mayor abundancia fueron Planorbidae y Tubificidae en el periodo de estudio.

La diversidad alfa para las zonas de muestreo mediante el Índice de Shannon es de 0 1.45 y de Simpson de 0 - 0.7 lo que significa una baja diversidad; la diversidad beta mediante el Índice de Jaccard registra una similaridad entre 15 y 100\%; mientras que el Índice de Morisita registra una homogeneidad entre 8 y $100 \%$, lo que indica que existe variación en el espacio y el tiempo.

La comunidad bentónica de la laguna de Piuray presenta una variación espacio temporal, en los meses de mayo a julio, debido a factores de profundidad, incidencia de luz y sustrato.

\section{Autor corresponsal:}

Katherine M. Cruz-Fernández Baca

Correo electrónico: kathycfb_gym@ @hotmail.com - ORCID 0000-0002-7607-2049

\section{REFERENCIAS BIBLIOGRÁFICAS}

Centro de Educación y Comunicación Guaman Poma de Ayala. (2013). Estudio del Plan de Gestión Integral de los Recursos Hídricos de la Microcuenca de la Laguna de Piuray, Diagnóstico Técnico. Cusco. Perú.

Clifford, H. F. (1991). Aquatic invertebrates of Alberta: An illustrated guide. Edmonton [Alta.]: University of Alberta Press, Alberta, Canadá. 
Karr, J. R. (1991). Biological integrity: a long-neglected aspect of water resource management. Ecology Applic. 1 p.66-84. doi: 10.2307/1941848.

Roldán, G. (1999). Los macroinvertebrados y su valor como indicadores de la calidad del agua. Rev. Acad. Colomb. Cienc. 23(88):375 - 387. 1999. ISSN 0370-3908.

Oscoz, J.; Galicia, D.; Miranda, R. (2011). Clave dicotómica para la identificación de macroinvertebrados de la cuenca del Ebro. Edita Confederación Hidrográfica del Ebro. Zaragoza, España

Rosas, J.; Miranda, G. (2014). Estructura temporal y espacial de las comunidades planctónicas de la laguna de Piuray - Chinchero - Cusco. Tesis para optar al Título profesional de Biólogo. UNSAAC. Cusco.

Anexo 1. Registro fotográfico

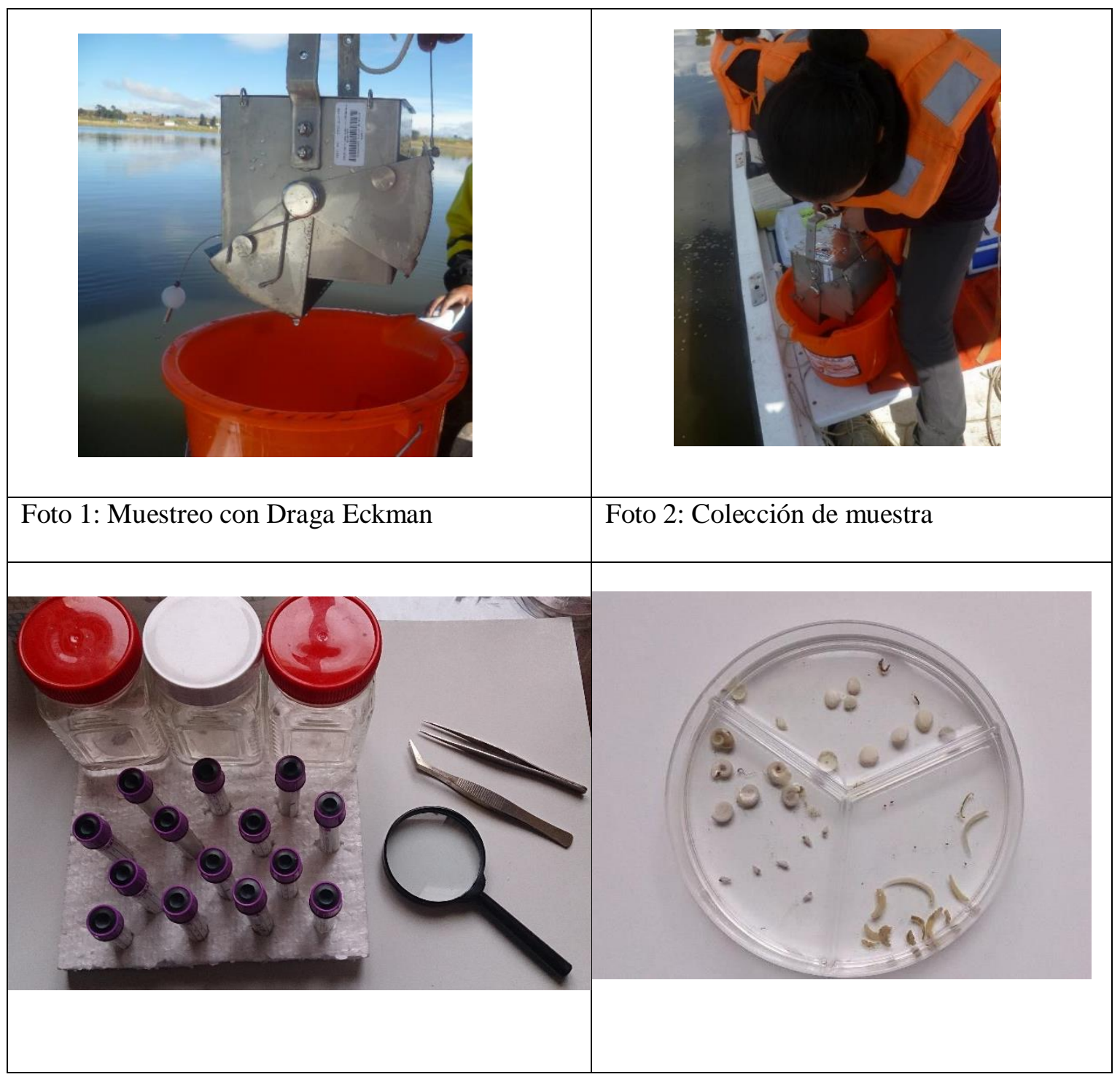




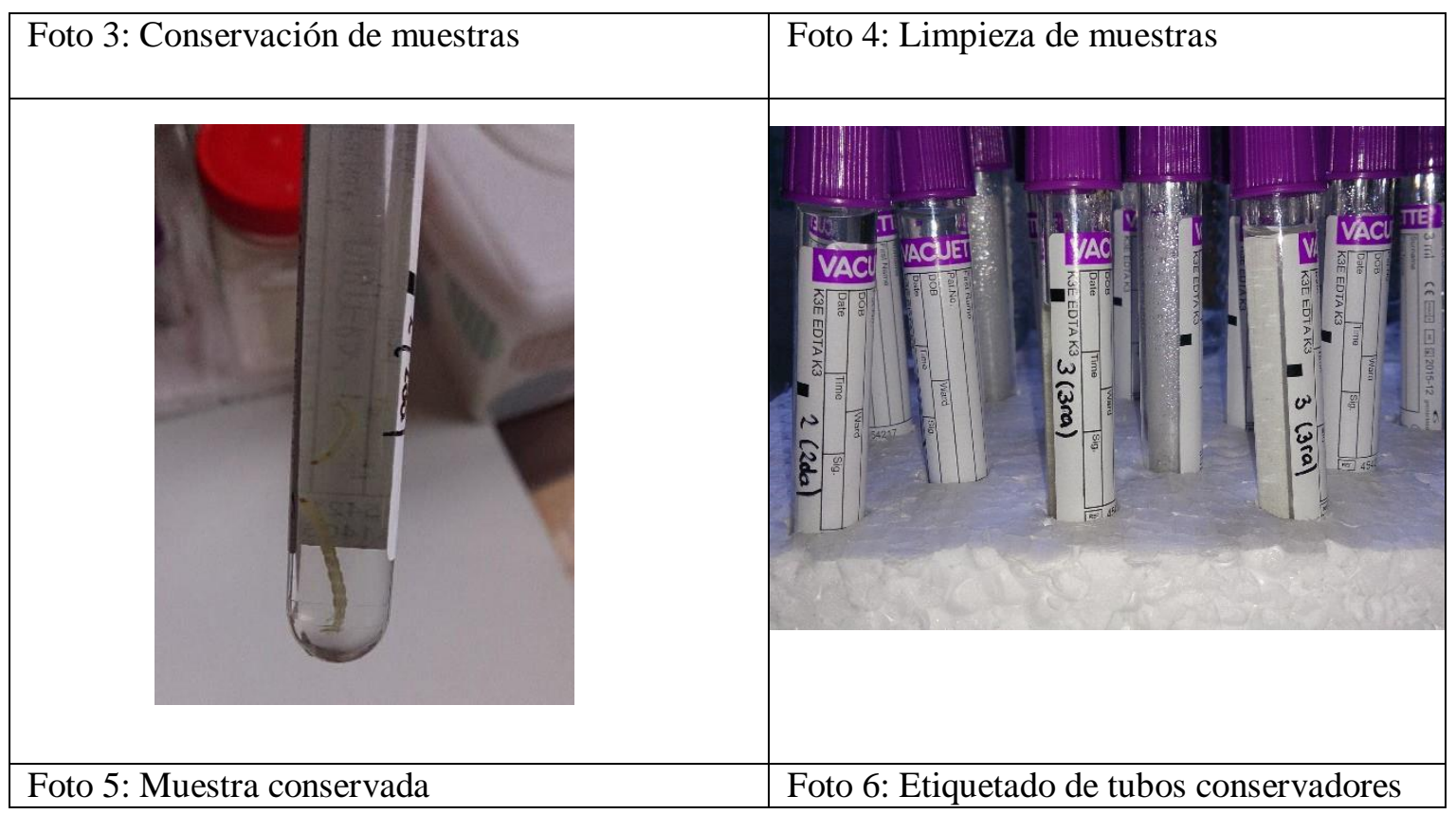

COMUNIDAD BENTONICA DE LA LAGUNA DE PIURAY

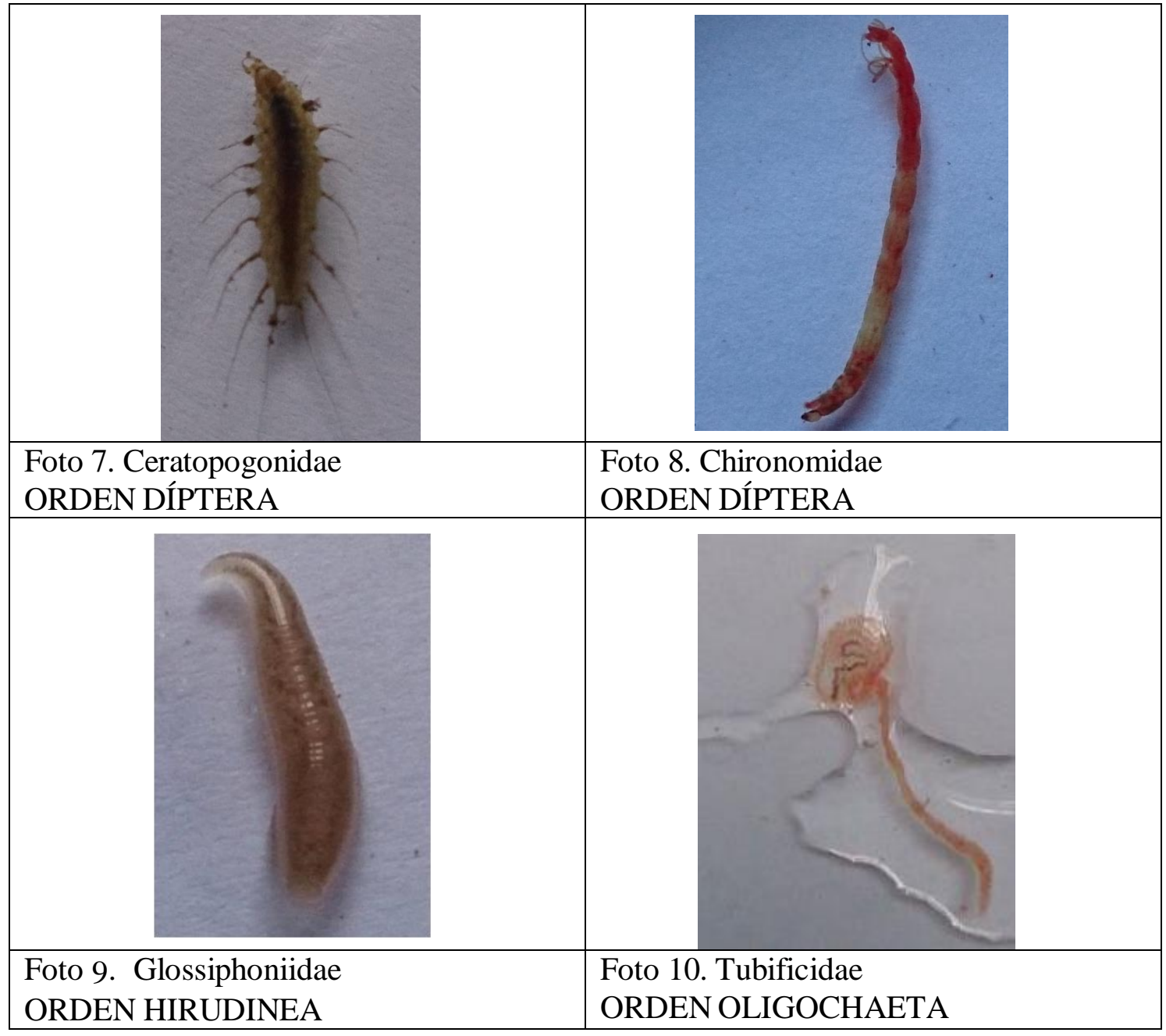




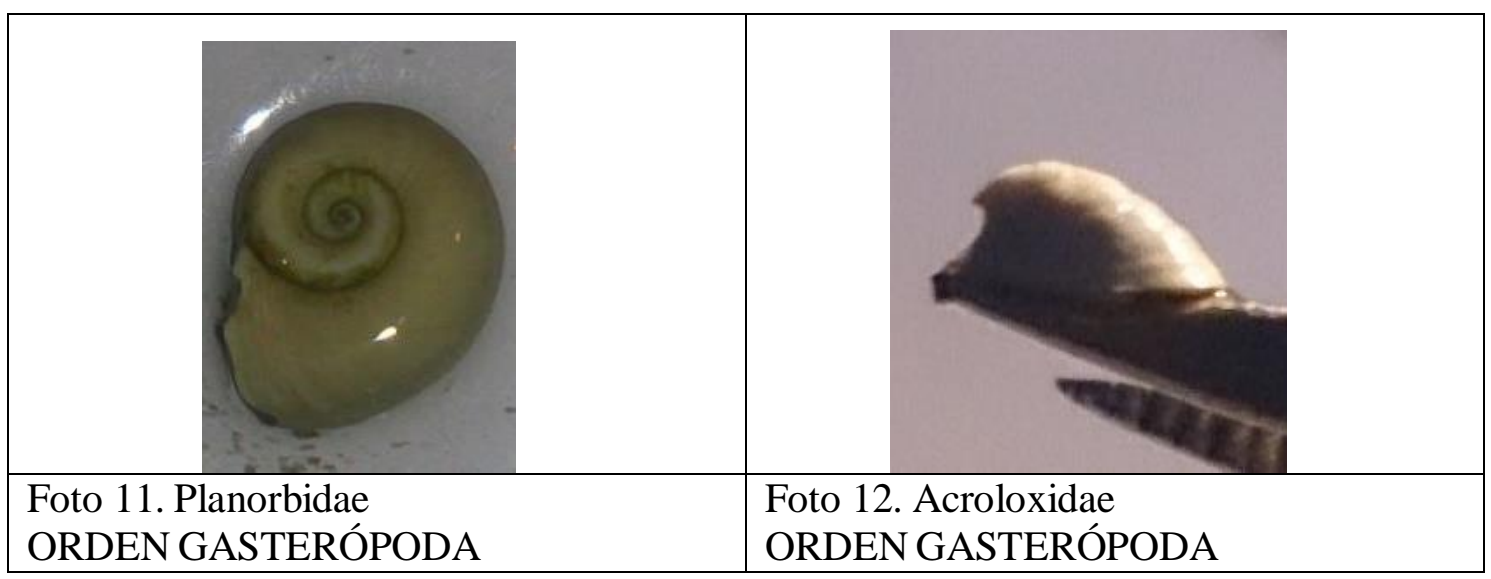

